\title{
An analysis of the triaxial apparatus using a mixed boundary three-dimensional discrete element model
}

\author{
L. CUI*, C. O’SULLIVAN† and S. O’NEILL
}

\begin{abstract}
The triaxial test is probably the most important fundamental laboratory test for geotechnical engineers. The objective of the study presented here was to gain insight into the micro-scale interactions experienced by particles during a triaxial test using the distinct element method (DEM). To achieve this objective, a novel DEM modelling environment to simulate triaxial tests on ideal granular materials was implemented, as described here. In this test environment, both the stress conditions and boundary conditions in the physical tests can be accurately reproduced. This approach was quantitatively validated by simulating a number of physical triaxial tests on specimens of steel spheres. A comparison between the circumferential periodic boundaries used here and the circumferential rigid boundaries used by other researchers emphasises the significance of maintaining a continuous particle-particle contact network orthogonal to the major principal stress direction. Results of an analysis of the micro-scale response in a triaxial simulation are analysed in detail, including the stresses, the distribution of contact forces, the evolution of fabric, the distribution of local strains, and the particle rotations. Significant non-uniformities in the stresses, strains and contact fabric were observed.
\end{abstract}

KEYWORDS: laboratory tests; model tests
Le test triaxial est probablement le test de laboratoire fondamental le plus important pour les ingénieurs géotechniciens. L'objectif de l'étude présentée dans cet article était de permettre une meilleure compréhension des interactions microscopiques subies par les particules lors d'un test triaxial utilisant la méthode d'élément distinct (MED). Pour ce faire, un nouvel environnement de modélisation MED visant à simuler des tests triaxiaux sur des matériaux granulaires idéaux a été mis en oeuvre, ainsi que cela est décrit de l'article. Dans cet environnement expérimental, il est possible de reproduire de façon précise les conditions de contrainte et les conditions de limite prévalant dans les tests physiques. Cette approche a été quantitativement validée en simulant un certain nombre de tests triaxiaux physiques sur des spécimens de sphère en acier. Une comparaison entre les limites périodiques circonférentielles utilisées ici et les limites périodiques circonférentielles utilisées par d'autres chercheurs a permis d'établir clairement la nécessité de maintenir un réseau de contact particule/particule continu orthogonal dans la direction de la contrainte principale majeure. Les résultats d'une analyse de la réponse microscopique lors d'une simulation triaxiale sont analysées en détails, notamment les contraintes, la distribution des forces de contact, l'évolution de la structure, la distribution des contraintes locales et les rotations de particules. Des nonuniformités significatives ont été observées pour tensions, les contraintes et la structure de contact.

\section{INTRODUCTION}

The triaxial test is probably the most commonly used geotechnical element test to determine soil response characteristics. Given the extensive use of triaxial testing in both geotechnical research and practice, it is important for geotechnical engineers to appreciate fully the details of material response in this apparatus. In recognition of this fact, there have been significant advances in instrumentation to make 'local' measurements during triaxial tests. For example, to measure local axial strains, LVDTs can be attached to the sample (Cuccovillo \& Coop, 1997), inclinometers can be mounted on the sample (Jardine et al., 1984), and Hall effect transducers can also be used (Clayton \& Khatrush, 1986). In the orthogonal direction, radial strain belts may be used (e.g. Klotz \& Coop, 2002). Researchers have also used image analysis to look at the distribution of particle displacements (and hence strains) along the exterior of the sample (e.g. Rechenmacher \& Finno, 2004; Sachan et al., 2006). All of

Manuscript received 4 July 2006; revised manuscript accepted 12 September 2007.

Discussion on this paper closes on 1 June 2008, for further details see p. ii.

* School of Architecture, Landscape \& Civil Engineering, University College Dublin, Ireland.

$\dagger$ Department of Civil and Environmental Engineering, Imperial College London, UK (formerly Department of Civil Engineering, University College Dublin).

$\$$ Formerly University College Dublin. these methods focus on the local response on the exterior of the sample. Attempts to explore what is happening inside the specimen include the work of Jang \& Frost (2000), who examined sections through resin-injected samples, and Otani et al. (2000), who used micro X-ray tomography to study the evolution of localisations in triaxial tests. A significant amount of information about soil response in the triaxial test has been obtained using these advanced measurement techniques. However, even if further improvements are made in the area of instrumentation, measurement of certain parameters including the interparticle forces will remain intractable.

The distinct element method (DEM), as proposed originally by Cundall \& Strack (1979), is a method of numerical analysis for granular materials (including soils) that explicitly models the interaction of individual particles, and allows information about the particle displacements, rotations and interactions to be monitored during simulations of element tests and subsequently analysed. While a DEM model uses relatively simple particle geometries, it is a useful approach to understand better the micro-scale interactions in the triaxial tests. A number of DEM simulations of triaxial tests have been performed by earlier researchers, including Thornton (2000), O'Sullivan et al. (2003), Ng (2004), and Tsunekawa \& Iwashita (2001). The boundary conditions used in these earlier studies were periodic boundaries, rigid boundaries, hydrostatic boundaries and flexible membrane boundaries respectively. Most of these earlier studies did not accurately replicate the physical test boundary conditions, that is, the conditions where a cylindrical specimen is enclosed within a 
latex membrane and a confining pressure is applied to the specimen through the membrane. To model accurately the granular material response in the triaxial apparatus, and to attain quantitative agreement between the specimen response in the simulation and the physical test, it is important to reproduce the test boundary conditions accurately.

This paper presents a mixed boundary approach to simulate triaxial tests, and outlines the implementation of this approach in a 3DDEM code. A series of DEM simulations of laboratory tests on specimens of randomly packed spheres are then described to validate the proposed test environment. The sensitivity of the macro-scale response to the friction coefficient and the circumferential boundary conditions is then analysed. The remaining sections of the paper present a detailed analysis of the particle-scale parameters, including an examination of the contact forces, the contact fabric, the coordination number, the particle displacements, the local strains and the particle rotations.

\section{IMPLEMENTATION OF THE MIXED BOUNDARY TEST ENVIRONMENT}

The DEM simulations described in this paper used a DEM code called 3DDEM, developed by O'Sullivan (2002) and Cui (2006) based on the ELLIPSE3D code (Lin \& Ng, 1997). In the simulations the top and bottom boundaries were modelled using planar rigid wall boundaries. The lateral boundary conditions were more complex and are described here.

\section{Implementation of periodic boundaries}

As the triaxial apparatus is cylindrical, and symmetric around a central axis, it should be sufficient to model one 'slice' of the specimen and thus gain significant computational efficiency. Note that, in a similar manner, the computational cost of threedimensional finite element analyses is greatly reduced if an axisymmetric framework is adopted (e.g. Potts \& Zdravkovic, 1999). Other authors (e.g. Morchen \& Walz, 2003) have proposed that, for such axisymmetric analyses, the slice should be bounded by rigid vertical boundaries. However, in the current study emphasis was placed on maintaining a continuous internal system of particle-to-particle contacts throughout the specimen. To this end, a new boundary condition for threedimensional axisymmetric analyses, called 'circumferential periodic boundaries', has been proposed by the authors. These circumferential periodic boundaries, illustrated in Fig. 1(a), are conceptually similar to the rectangular periodic boundaries that are widely used in DEM simulations (e.g. Thornton, 2000). Particles with their centres moving outside one circumferential boundary $(\mathrm{O}-\mathrm{a})$ are reintroduced at a corresponding location along the other circumferential boundary (O-b) (Fig. 1(b)). Contact forces can develop between the particles close to each periodic boundary and particles along the other periodic boundary. These forces are calculated by using a rotation tensor when calculating the interparticle distances (Fig. 1(c)).

In theory, should the proposed approach to modelling axisymmetric problems work, it can be implemented for an arbitrary inclination of the periodic boundaries to each other $(\theta$, as illustrated in Fig. 1(b)). In the current (initial) study, however, orthogonal circumferential boundaries are selected to simplify the contact force calculations along the periodic boundaries (as the algorithm for contact detection in the current code uses a series of rectangular boxes on a Cartesian grid). The specimen is located with its vertical central axis coinciding with the $z$-axis; the $x$ - and $y$-axes then form a periodic boundary pair. The movement of a particle along one periodic boundary can be mapped to the other periodic boundary by an orthogonal rotation in the $x-y$ plane, and the rotation tensor $T$ is given by

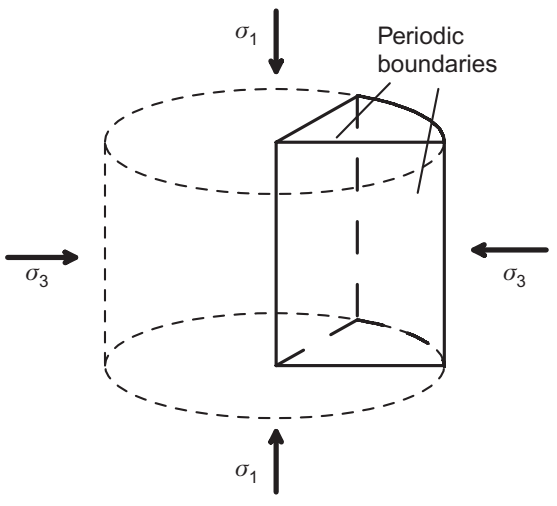

(a)

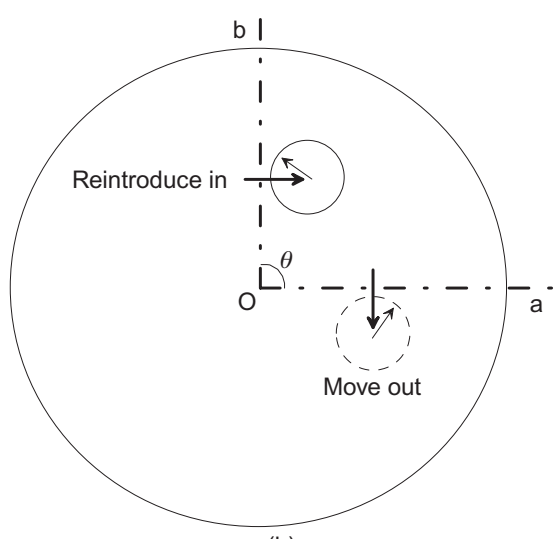

(b)

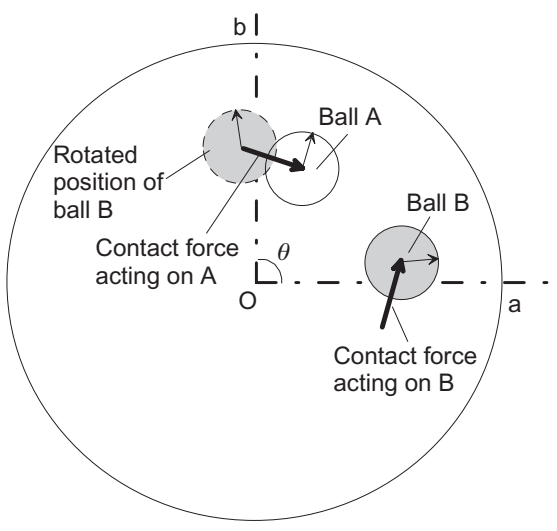

(c)

Fig. 1. (a) Schematic illustration of periodic boundaries; (b) illustration of reintroduced particles; (c) illustration of contact considerations

$$
\left(\begin{array}{l}
x^{\prime} \\
y^{\prime}
\end{array}\right)=T\left(\begin{array}{l}
x \\
y
\end{array}\right)=\left(\begin{array}{cc}
\cos \theta & -\sin \theta \\
\sin \theta & \cos \theta
\end{array}\right)\left(\begin{array}{l}
x \\
y
\end{array}\right)
$$

where $x^{\prime}, y^{\prime}$ are the coordinates after rotation, $x, y$ are the coordinates before rotation, and $\theta$ is the angle between the current periodic boundary and its partner periodic boundary (with anticlockwise rotation being positive). In the implementation of these boundaries, special care must be taken regarding particles that are located close to the origin. If a particle protrudes from both boundaries $(\mathrm{O}-\mathrm{a}$ and $\mathrm{O}-\mathrm{b})$, then forces along both periodic boundaries must be considered. Furthermore, if the particle centroid is located exactly along the $z$-axis, then the particle cannot move in the horizontal $(x-y)$ plane. A more detailed description of the implementation of these circumferential periodic boundaries is provided by Cui (2006). 


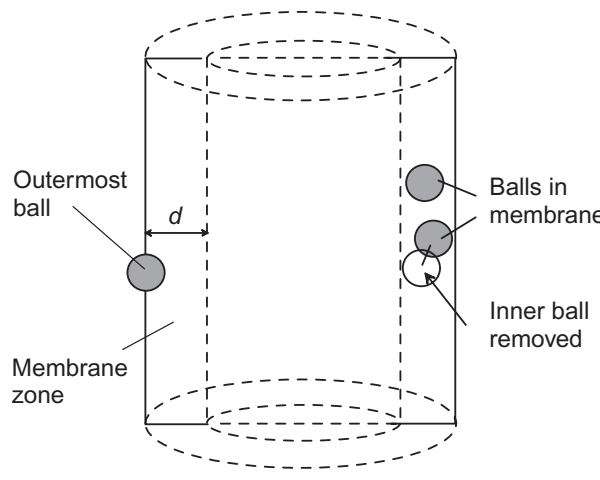

Fig. 2. Schematic diagram to illustrate identification of membrane balls

Implementation of stress-controlled cylindrical membrane

A key consideration in simulating physical triaxial tests is modelling the flexible latex boundary. Various numerical 'membranes' have been proposed in the literature, in both two and three dimensions, and there are some differences between them. In the current work the 'membrane spheres'-that is, those spheres that would contact the membrane in the physical tests - are identified by considering the spheres close to the edge of the sample (see Fig. 2). A sphere is considered to participate in the numerical membrane if none of the contact forces associated with this sphere has a contact normal orientated radially outwards. A force is then applied to each of these spheres so that the average stress applied along the boundary equals the confining pressure measured in the laboratory. Note that while the deviator stress $\left(\sigma_{1}-\sigma_{3}\right)$ in the physical test is calculated from the external load cell, the stress $\sigma_{1}$ in the simulation is measured by considering the average vertical stress acting on the top and bottom rigid boundaries.

To calculate the required forces, the polar coordinates of the boundary particles are projected onto a plane $S^{\prime}$, which is obtained by unfolding the cylindrical surface $S$ going through the centre of the membrane zone (zone containing all the membrane particles). Then a Voronoi diagram is generated on the rectangular surface $S^{\prime}$. (For a given set of points $p_{i}$, a Voronoi diagram divides the space into polygonal regions $V_{i}$ in such a way that the region $V_{i}$ is the space closer to $p_{i}$ than to any other point (Shewchuk, 1999).) The force to be applied to each 'membrane sphere' is calculated by multiplying the confining pressure and the area of the Voronoi polygon surrounding the centroid of each sphere.

The challenge when generating the Voronoi diagram for the current application is the necessity to cover the entire rectangular projection area of surface $S^{\prime}$ using the Voronoi diagram. The Voronoi diagram around the spheres along the top and bottom boundaries and the periodic boundaries may exceed the boundaries of the projection area, and voids may also exist along those boundaries (refer to Fig. 3(a)). To ensure the Voronoi diagram only covers the entire area without leaving any voids, the following was implemented.

(a) Additional 'virtual' points are introduced just above the top boundary and just below the bottom boundary (Fig. 3(b)).

(b) Additional virtual points are also introduced along the periodic boundaries. If a particle is close to the periodic boundary, an additional point is introduced outside the other periodic boundary protruding the same distance (Fig. 3(b))

(c) The Voronoi diagram is then generated based on the set of points including both the real centres of membrane spheres and the additional virtual points as described above.

(d) Finally, if any vertex of the Voronoi polygon is outside

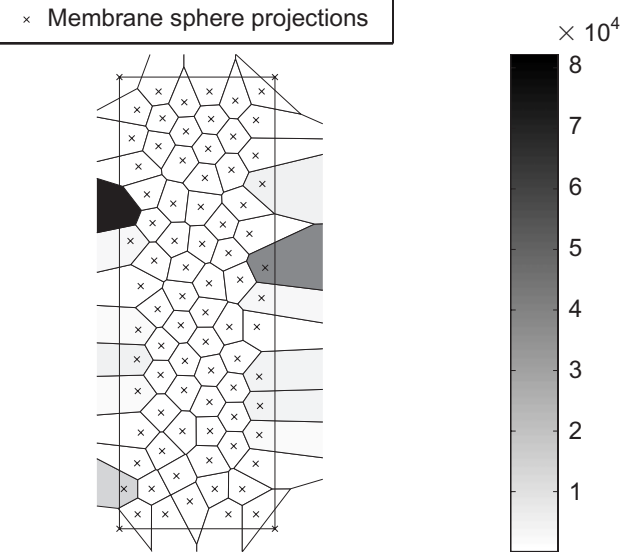

(a)

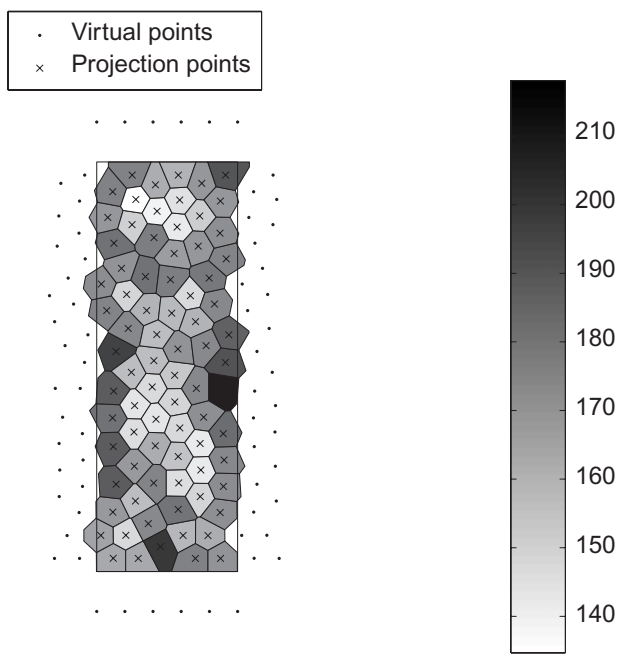

(b)

Fig. 3. Schematic diagram of Voronoi diagram and external forces: (a) before modification; (b) following modification

the top or bottom boundaries, this vertex is moved onto the relevant boundary.

To illustrate this technique, a representative figure showing the Voronoi diagrams for a typical membrane and the magnitude of the external force applied on each sphere is shown in Fig. 3(b). The difference between the summation of the Voronoi polygon areas and the projection area $\left(S^{\prime}\right)$ was typically lower than $0 \cdot 1 \%$ for all the simulations considered here.

It is important to recognise that, during the simulation, the coordinates of the spheres and consequently the geometry of the membrane will change. Therefore the list of spheres in the membrane and the calculated applied forces on those spheres are updated if the accumulated displacement of any sphere exceeds a user-specified limit or the relative difference between the internal radial stress and the confining stress is larger than a specified tolerance.

\section{VALIDATION OF THE TEST ENVIRONMENT Preliminary theoretical validation}

Following implementation of these boundaries, a series of preliminary validation simulations was performed to assess their performance. These preliminary simulations considered specimens of uniform spheres with a face-centred-cubic (FCC) packing configuration, as analytical estimates of the stress ratio at failure for such specimens can be developed (Thornton, 1979). A quantitative match between the simula- 
tion results and the analytical results was achieved (within $5 \%$ error). A description of the preliminary validation was presented in Cui \& O'Sullivan (2005).

\section{Comparison of physical triaxial tests and equivalent DEM simulations}

Further validation was achieved using a series of laboratory triaxial tests performed on specimens of steel spheres. There are obvious differences between real sands and steel spheres; however, by coupling DEM simulations with physical tests on this 'ideal soil' in this way, the geometry of the material can be accurately replicated in the DEM model, facilitating quantitative validation of the model. Conclusions about the micro-scale response of the material in the triaxial apparatus can then be made with confidence using the DEM simulation data (e.g. Cui \& O'Sullivan, 2006).

All of the laboratory tests were performed on specimens of three-dimensional assemblies of Grade 25 chrome steel spheres under vacuum confinement in a triaxial cell. The vacuum confined approach was also used by O'Sullivan (2002) in laboratory plane strain tests and triaxial tests. The sphere material density is $7.8 \times 10^{3} \mathrm{~kg} / \mathrm{m}^{3}$, the shear modulus is $7.9 \times 10^{10} \mathrm{~Pa}$, and the Poisson's ratio was $0 \cdot 28$. Two different types of specimen were used: spheres with radii of $2.5 \mathrm{~mm}$ (monodisperse specimens), and mixture of spheres with radii of $2 \mathrm{~mm}, 2.5 \mathrm{~mm}$ and $3 \mathrm{~mm}$ with a 1:1:1 mix (polydisperse specimens). Each specimen was created using dry pluviation with a funnel to minimise the drop height. The prepared specimens were $101 \mathrm{~mm}$ in diameter and $203 \mathrm{~mm}$ high. The specimens were compressed at an axial strain rate of $0.0083 \%$ / $\mathrm{s}$ by raising the bottom boundary (i.e. the tests were strain controlled). The vertical force applied to the specimen was measured on the stationary top boundary. The vacuum confining pressure in all the laboratory tests was set to $80 \mathrm{kPa}$. Full details of these tests can be found in O'Neill (2005).

For the DEM simulations the particle coordinates were initially obtained using an algorithm that generates random dense assemblies of non-contacting spheres (Jodrey \& Tory, 1985). The required void ratios were obtained by gradually expanding the radii and controlling the confining pressure. It was found that using a confining pressure of $3000 \mathrm{kPa}$ ensured that the void ratios of the numerical specimens were close to the laboratory specimens. Using this approach, two cylindrical quadrant specimens with 3852 and 3848 spheres were obtained, with radii of $50 \mathrm{~mm}$ and heights of $200 \mathrm{~mm}$. These specimens correspond to full cylindrical specimens of 15408 and 15392 particles with void ratios of 0.615 and 0.617 respectively. The laboratory test specimens contained between 15382 and 15420 spheres, with void ratios of 0.615 and 0.612 respectively. The discrepancy between the confining pressure used in the physical tests and the confining pressure used in the DEM simulations is acknowledged. However, for the specimen generation approach used, the authors could control either the void ratio alone or the average stress alone, with the other parameter being a resultant value. In the simulations presented here, we chose to control the void ratio as the specimen response is quite sensitive to the void ratio, and the applied stresses are the stresses under which the specimen came into equilibrium for the specified void ratio. Using this approach, two specimens containing a mixture of spheres with radii of $2 \mathrm{~mm}, 2.5 \mathrm{~mm}$ and $3 \mathrm{~mm}$ with a $1: 1: 1 \mathrm{mix}$ were also obtained for comparison with equivalent physical tests.

During shearing, the specimen was compressed by moving the top boundary at a rate of $0.0083 \% / \mathrm{s}$. The average intersphere friction coefficient of 0.096 measured by O'Sullivan et al. (2004) for equivalent spheres was assumed in the current study. The friction coefficient between the particles and the top and bottom plates used in the laboratory tests was measured using a series of tilt tests, as described by Cui (2006), giving a value of $0 \cdot 228$. The input parameters for the DEM simulations are equivalent, apart from the differences in the density value used. Density scaling is commonly used to reduce the computational cost associated with DEM simulations for quasi-static analyses (e.g. Thornton, 2000; O'Sullivan et al., 2004).

In a triaxial test, the cross-sectional area increases owing to the increasing axial strain $\varepsilon_{\mathrm{a}}$. An area correction is typically adopted to calculate the cross-sectional area. In the laboratory triaxial tests a vacuum confinement was used, and there was no water in the triaxial cell. Therefore it was impossible to measure the change in the specimen volume and the cross-sectional area by measuring the change in the volume of the cell water. In the calculations of the laboratory test results, the axial stress was calculated by the boundary force divided by the original cross-sectional area. To facilitate a direct comparison with the laboratory tests, the axial stresses in the DEM simulations were first calculated without area correction as for the physical tests. Then the axial stress was re-calculated using the boundary force divided by the average cross-sectional area throughout the specimen at the corresponding axial strain $\varepsilon_{\mathrm{a}}$. As the boundary stresses on the top boundary and the bottom boundary

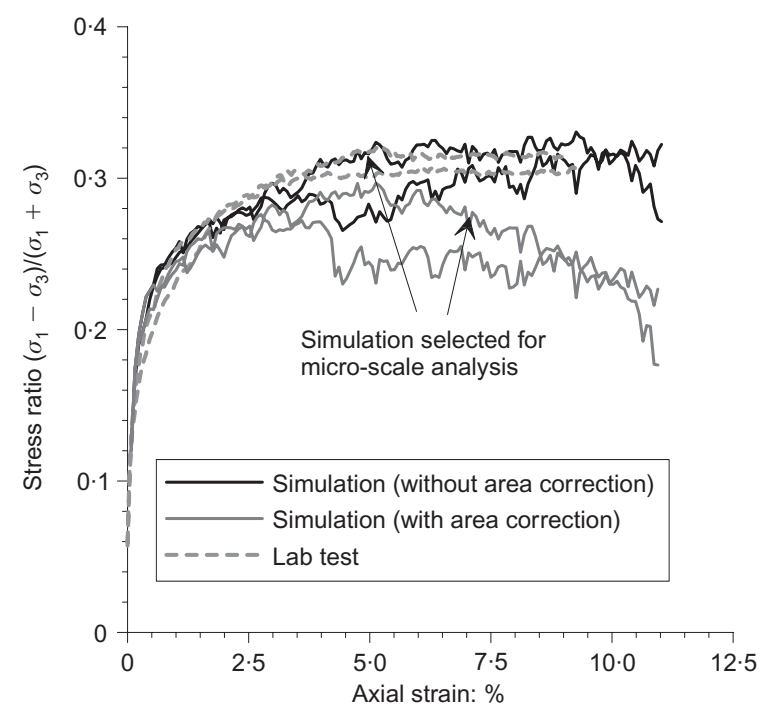

(a)

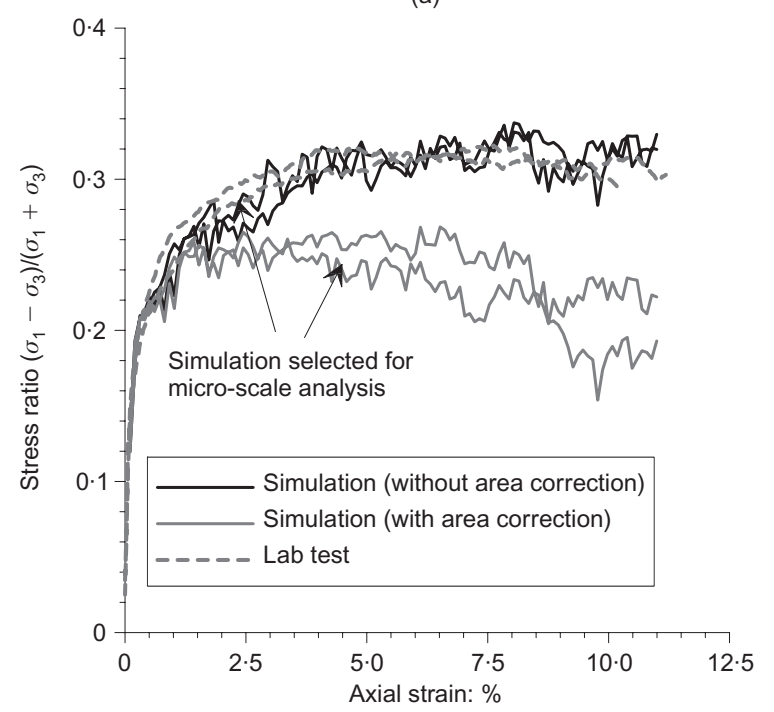

(b)

Fig. 4. Macro-scale comparison of DEM simulation results and physical tests: (a) monodisperse specimens; (b) polydisperse specimens 
are equivalent, only the stress on the stationary bottom boundary is considered in the analyses.

The variation in the stress ratio, $\left(\sigma_{1}-\sigma_{3}\right) /\left(\sigma_{1}+\sigma_{3}\right)$, with axial strain for the laboratory tests and the DEM simulations is illustrated in Fig. 4. Note that the DEM results were calculated both with application of an area correction and without use of an area correction. Fig. 4(a) illustrates the macro-scale response for the monodisperse specimen and Fig. 4(b) shows the macro-scale response of the polydisperse specimen. The normal forces along the top and bottom boundaries were monitored throughout the simulations; they were consistently approximately equal in magnitude, confirming quasi-static conditions.

As illustrated in Fig. 4, the stress-strain response of DEM simulations without area correction closely matches the response of the laboratory tests, including the initial stiffness and large-strain response. These results quantitatively validate the mixed test environment for the triaxial test. The post-peak strain-softening is not obvious in either the laboratory tests or the corresponding simulations, where the original area is used to calculate $\sigma_{1}$. In contrast, where the actual average area is considered, there is an obvious strain-softening.

The angle of shearing resistance $\left(\phi^{\prime}\right)$ was calculated as sin $\phi^{\prime}=\left(\sigma_{1}-\sigma_{3}\right) /\left(\sigma_{1}+\sigma_{3}\right)$. In the physical tests the two monodisperse specimens yielded peak angles of shearing resistance $\left(\phi_{\text {peak }}^{\prime}\right)$ of $17.9^{\circ}$ and $18.7^{\circ}$, for initial void ratios $\left(e_{0}\right)$ of 0.612 and 0.615 respectively. In the corresponding DEM simulations both the specimens reached a $\phi_{\text {peak }}^{\prime}$ value of $19 \cdot 2^{\circ}$ (without any area correction), for $e_{0}$ values of 0.615 and 0.617 . Where the area correction was used, the two DEM specimens yielded $\phi_{\text {peak }}^{\prime}$ values of $17 \cdot 4^{\circ}$ and $16 \cdot 1^{\circ}$. The polydisperse

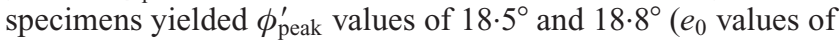
0.603 and 0.604 ) in the physical tests, whereas the DEM simulations obtained $\phi_{\text {peak }}^{\prime}$ values of $20 \cdot 0^{\circ}$ and $20 \cdot 1^{\circ}\left(e_{0}\right.$ values of 0.604 ) where no area correction was considered in stress calculation. Upon applying an area correction, the $\phi_{\text {peak }}^{\prime}$ values for the simulations were $15 \cdot 9^{\circ}$ and $16 \cdot 2^{\circ}$ respectively. The $\phi_{\text {peak }}^{\prime}$ values without area correction in the simulations are slightly higher than the values in the physical tests. Both the physical tests and the simulations exhibited slight scatter.

A comparison of the monodisperse and polydisperse specimens shows that the $\phi_{\text {peak }}^{\prime}$ value of the polydisperse specimen without area correction is about $1.0^{\circ}$ higher than that of the monodisperse specimen, and the $\phi_{\text {peak }}^{\prime}$ value of the polydisperse specimen with area correction is lower than that of the monodisperse specimen. This trend arose because the average cross-sectional area of the polydisperse specimen was greater during the simulation than that of the monodisperse specimen: that is, the polydisperse specimens exhibited greater radial dilation than the monodisperse specimens. We note that the polydisperse specimens have a slightly lower void ratio than the monodisperse specimens. A detailed analysis of the micro-scale response of both specimen types is explored below.

\section{Sensitivity analyses to surface friction}

A number of additional triaxial simulations were performed on a single representative monodisperse specimen, as indicated in Fig. 4(a), to explore the sensitivity of the macro-scale specimen response to the friction coefficient. The results are illustrated in Fig. 5. It can be observed from Fig. 5(a) that the initial stiffness increased with increasing interparticle friction coefficient $\left(f_{\mathrm{bb}}\right)$ values, while no obvious variation in the initial stiffness can be observed from Fig. 5(b) for various particle-boundary $\left(f_{\text {bd }}\right)$ friction coefficients. This trend differs from the findings by Rowe \& Barden (1964) and Frost \& Yang (2003) in their laboratory tests, where the initial stiffness with frictionless ends is

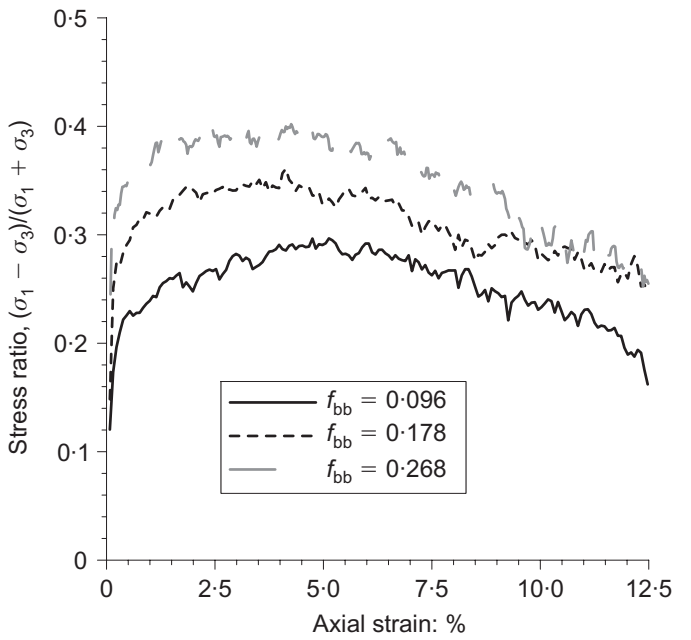

(a)

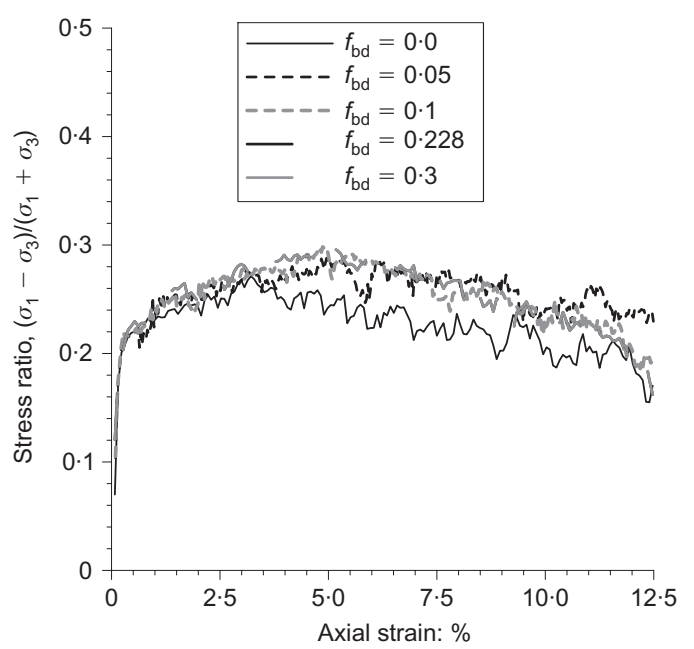

(b)

Fig. 5. Sensitivity of macro-scale response to: (a) interparticle friction coefficient $f_{\text {bb }}\left(f_{\text {bd }}=0 \cdot 228\right)$; (b) particle/boundary friction coefficient $f_{\mathrm{bd}}\left(f_{\mathrm{bb}}=\mathbf{0 \cdot 0 9 6}\right)$ (monodisperse specimen)

smaller than the stiffness with frictional ends. It is shown that the peak stress ratio of the specimen increases moderately as the $f_{\mathrm{bb}}$ values increase. The stress ratio increases slightly as the $f_{\mathrm{bd}}$ value increases from $0 \cdot 0$ to $0 \cdot 1$, and then remains almost constant at higher $f_{\text {bd }}$ values.

As would be expected, the particle/boundary friction values influenced the deformed shape of the specimen and, as a consequence, the average cross-sectional area. As illustrated in Fig. 6(a), a 'bulge' of the specimen was observed near the base boundary for the 'smooth' case with $f_{\mathrm{bd}}=0$. A similar bulge along the end of specimen was also observed by Rowe \& Barden (1964) in their physical freeended triaxial test. The constraint of the boundary increases as the $f_{\text {bd }}$ value increases. The radial dilation of the specimen was distributed nearly uniformly for $f_{\mathrm{bd}}=0.05$, as shown in Fig. 6(b). As $f_{\text {bd }}$ was further increased, the 'bulge' of the specimen moved from the end of the specimen to the mid-height of the specimen, as shown in Fig. 6(c). The deformed shape of the specimen did not vary greatly when the $f_{\text {bd }}$ value increased above $0 \cdot 1$.

\section{Sensitivity of macro-scale response to circumferential} boundary condition

As noted above, some researchers (e.g. Morchen \& Walz, 2003) have used two rigid frictionless circumferential verti- 


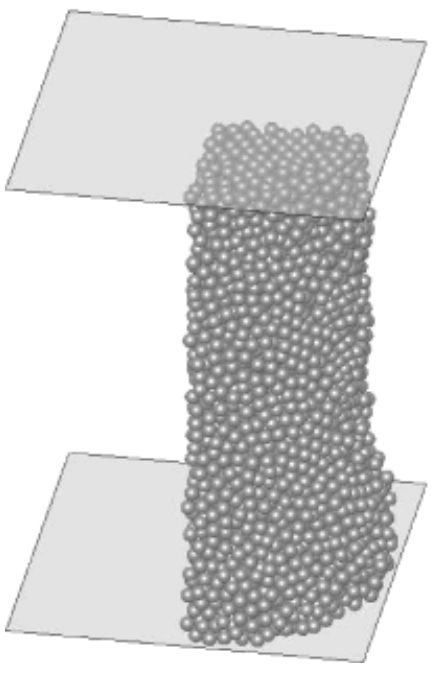

(a)

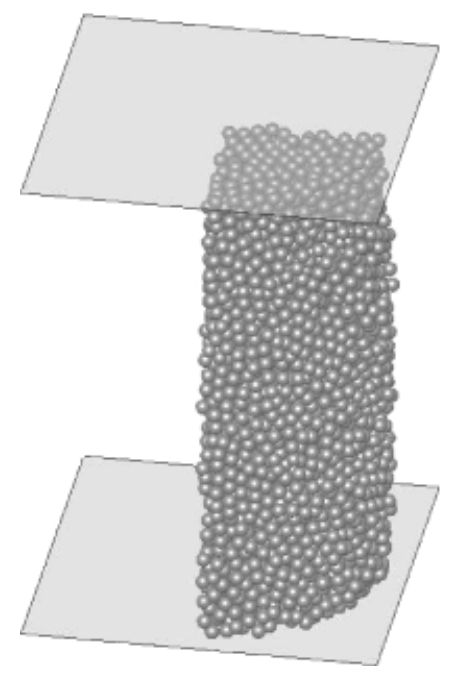

(b)

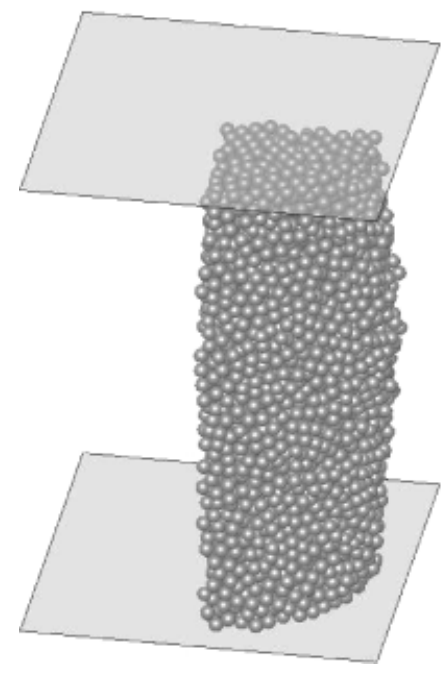

(c)

Fig. 6. Sensitivity of deformed specimen shape $\left(\varepsilon_{\mathrm{a}}=15 \cdot 3 \%\right)$ to particle/boundary friction coefficient (monodisperse specimen): (a) $f_{\text {bd }}=0 \cdot 0$; (b) $f_{\text {bd }}=0 \cdot 05$; (c) $f_{\text {bd }}=0 \cdot 1$

cal boundaries in their axisymmetric simulations to reduce the computational costs. Some simulations with both frictionless rigid boundaries and frictional rigid boundaries instead of periodic boundaries on monodisperse specimens were performed to explore the sensitivity of the response to the circumferential boundary condition. To obtain a symmetric deformation about the mid-height plane, all these specimens were compressed by moving both the top and base boundaries. A comparison of the simulation results obtained using rigid boundaries with those obtained using periodic boundaries is illustrated in Fig. 7 (with area correction applied in all cases).

As shown in Fig. 7, the replacement of the periodic boundaries by the frictionless rigid boundaries reduces the strength of the specimen greatly, with $\phi_{\text {peak }}^{\prime}=15 \cdot 2^{\circ}\left(\phi_{\text {peak }}^{\prime}\right.$ $=17 \cdot 4^{\circ}$ for periodic boundary conditions). However, the simulation results with frictional rigid boundaries yielded $\phi_{\text {peak }}^{\prime}=18 \cdot 1^{\circ}$ and $\phi_{\text {peak }}^{\prime}=20 \cdot 0^{\circ}$ for $f_{\text {bd }}=0.096$ and $f_{\text {bd }}=$ 0.228 respectively. The $\phi_{\text {peak }}^{\prime}$ value increases as the friction coefficient between the particles and the rigid boundaries increases. These two simulations overestimated the shear strength of the specimen. The shear strength obtained increases as the friction coefficient between the particle and the rigid boundary increases, so it could be argued that there might be an $f_{\text {bd }}$ value to obtain an equivalent $\phi_{\text {peak }}^{\prime}$ as measured in the physical tests. However, it would be difficult to determine this exact $f_{\text {bd }}$ value, and in any case it does not replicate the internal distribution of contact forces, as considered below. As discussed by O'Sullivan (2002), the number of particles in the specimen may influence the specimen response. The sensitivity of the circumferential boundary conditions was also considered for a monodisperse specimen containing 10000 spheres. The difference between these two boundary conditions did not decrease as the number of particles was increased. In the earlier study considering uniformly sized spheres with FCC packing (Cui \& O'Sullivan, 2005), a similar sensitivity to the circumferential boundary condition was observed.

The circumferential boundaries also influence the deformed specimen shape. In the frictionless case $\left(f_{\mathrm{bd}}=\right.$ $0.0)$ the deformed geometry resembles that observed in the laboratory, as illustrated in Fig. 8(a). However, in the

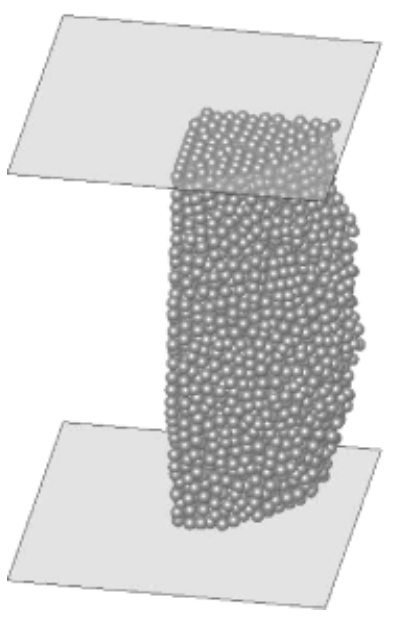

(a)

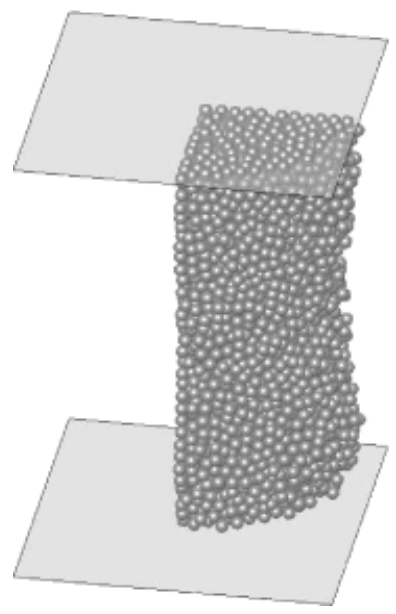

(b)
Fig. 8. Deformed specimen shape for monodisperse specimen with rigid circumferential boundaries $\left(\varepsilon_{\mathrm{a}}=15 \cdot 4 \%\right)$ : (a) $f_{\text {bd }}=$

$0 \cdot 0$; (b) $f_{\text {bd }}=0 \cdot 096$
Fig. 7. Sensitivity of specimen macro-scale response to circumferential boundary condition (monodisperse specimen) 
frictional case $\left(f_{b d}=0.96\right)$ the frictional forces between the particles and the rigid boundaries restricted the vertical motion of the particles near the top and bottom boundaries, as illustrated in Fig. 8(b). Consequently, these particles in these regions tended to move outwards, forming a 'bulge' in the specimen near the top and bottom boundaries.

\section{ANALYSIS OF PARTICLE-SCALE PARAMETERS}

A single representative simulation on a monodisperse specimen as indicated in Fig. 4(a) was selected to analyse the micro-scale response during the simulations.

\section{Stress analysis}

The average internal stresses in measurement regions 1,2 and 3 as illustrated in Fig. 9(a) were calculated. In many published DEM studies circular (for two-dimensional) or spherical (for three-dimensional) volumes are used to calculate the average internal stresses. However, the average stress $\left(\bar{\sigma}_{i j}\right)$ can be calculated by considering the total number of contacts $N_{\mathrm{c}}$ within an arbitrarily shaped volume $V$, using the formula

$$
\bar{\sigma}_{i j}=\frac{1}{V} \sum_{c=1}^{N c} l_{i}^{c} f_{j}^{c} \quad(i, j=x, y, z)
$$

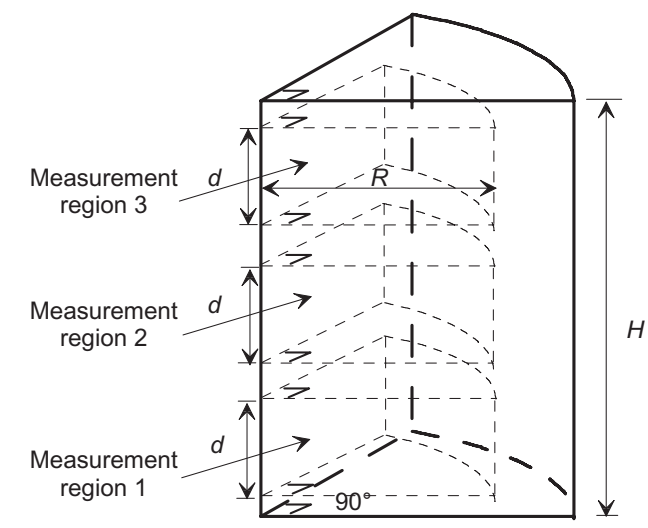

(a)

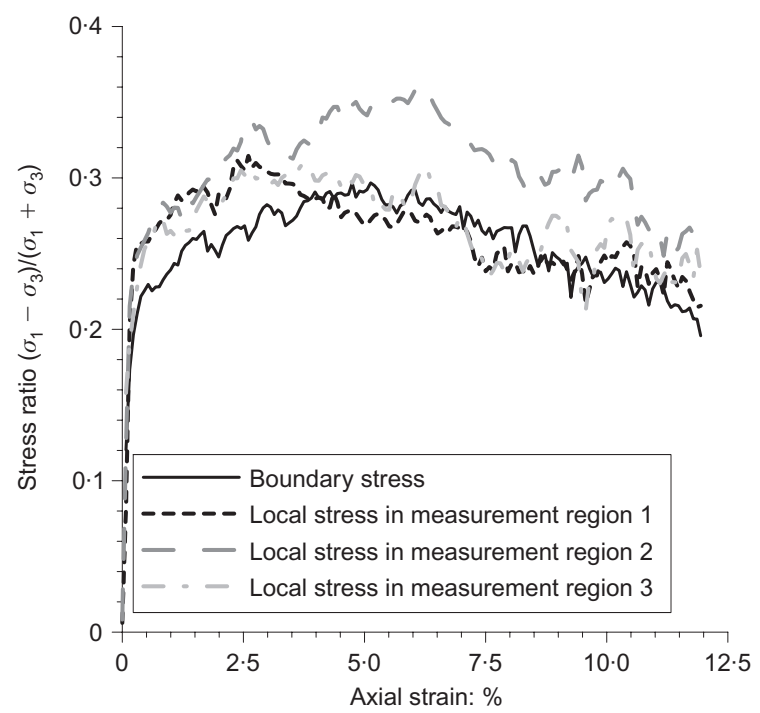

(b)

Fig. 9. Comparison of boundary stress and internal stresses for monodisperse specimen: (a) illustration of volumes used for internal stress calculation; (b) stress ratio against axial strain where $l_{i}^{c}$ is the branch vector (joining the centroids of two contacting spheres) and $f_{j}^{c}$ is the contact force vector at contact $c$. For the current study, each measurement region is a quadrant of a cylinder, reflecting the axisymmetric simulation environment. The regions considered here have their mid-height coordinates located at $30 \mathrm{~mm}, 80 \mathrm{~mm}$ and $130 \mathrm{~mm}$ respectively, and have heights $h$ of $40 \mathrm{~mm}$ and radii $R$ of $40 \mathrm{~mm}$. The calculated internal stresses in these measurement regions are plotted in Fig. 9(b). A clear nonuniformity of stress along the elevation was observed. The stress ratio in the mid-height region is higher than the other two regions and also higher than the stress as measured on the boundary, reflecting the non-uniformity of both $\sigma_{1}$ and $\sigma_{3}$. It is noted that the peak stress ratios are mobilised at different axial strains in the different regions. The nonuniformity of stresses along the ends is an effect of the end restraint caused by the frictional top and bottom boundaries. The stresses calculated from the boundary forces were smaller than the stresses measured internally, as the stresses within the specimen are non-uniform. The boundary stresses are calculated by considering the forces transmitted throughout the entire specimen, whereas the internal stresses consider only an inner region with radius of $40 \mathrm{~mm}$. As the stronger 'load-carrying' force chains appear to be concentrated towards the centre of the specimen, the stresses measured close to the specimen centre will exceed the overall average stresses. For geometric reasons, the void ratio will tend to be lower near the boundaries, and this will influence the tendency of the strong force chains to be concentrated towards the specimen centre.

Distribution and orientation of interparticle contact forces

While visualisation of the force chains in three dimensions is non-trivial, some insight into the material response can be gained by looking at the plots of the interparticle contact forces. In order to explore the spatio-temporal variation in the contact forces of individual particles, two orthogonal views of the evolution of the contact force network for the simulation of a specimen of monodisperse spheres are plotted. To explore the variation of the distribution of the contact forces along the elevation, three horizontal segments of the specimen, as shown in Fig. 10, are considered in the plan $(x-y)$ view plots. As the specimen response is symmetric about the mid-height, the three segments selected are located in the lower half of the specimen.

The contact force networks at $\varepsilon_{a}$ values of $6.2 \%$ (around peak) and $12 \cdot 3 \%$ (post peak) are both shown in Fig. 11.

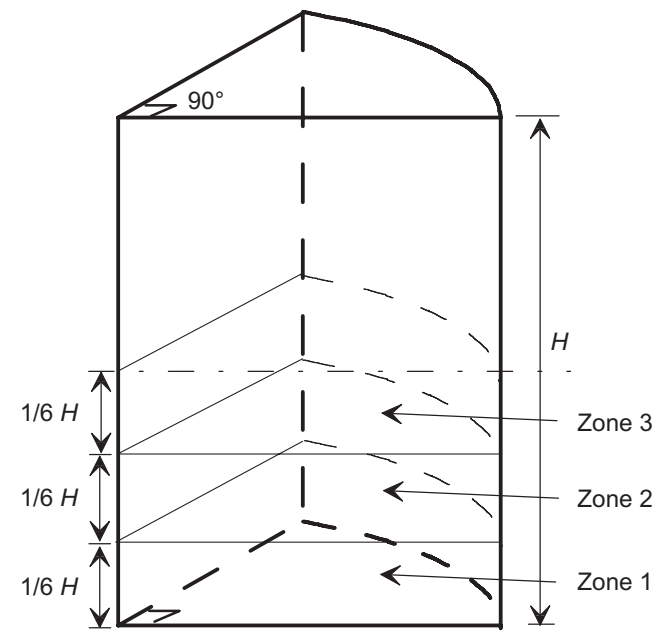

Fig. 10. Schematic diagram of three zones selected for detailed analysis of interparticle contact forces 

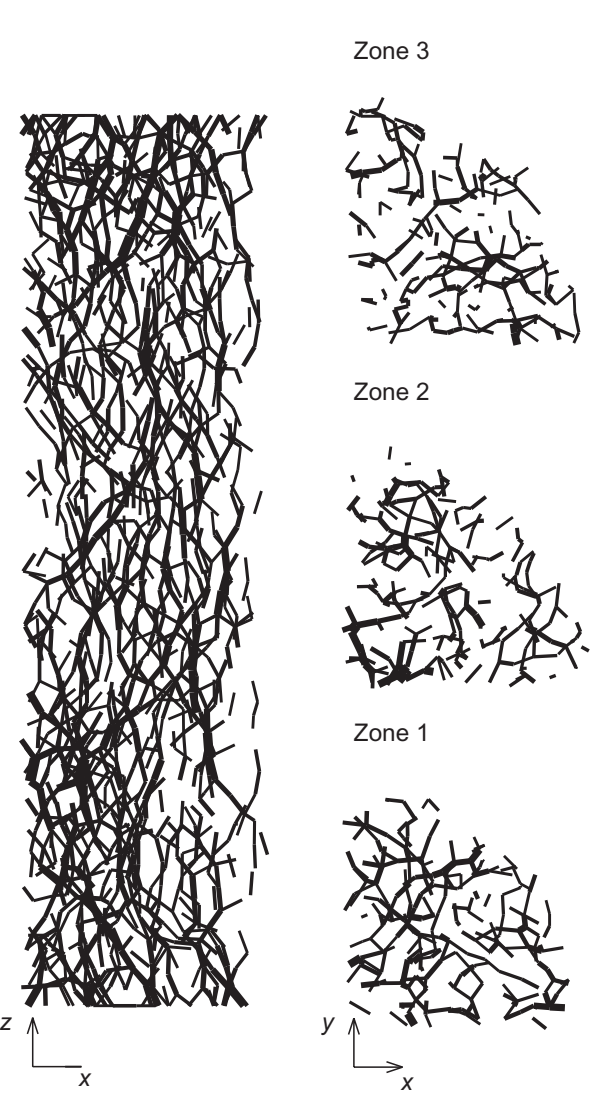

Zone 1

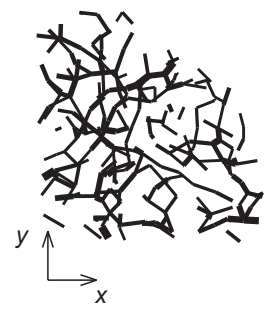

(a)
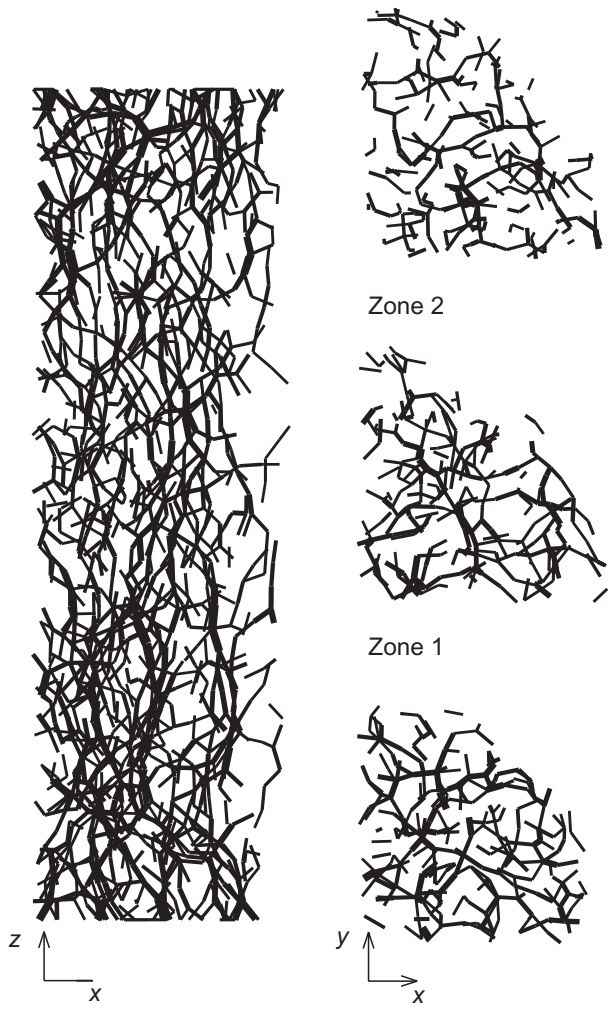

Zone 2

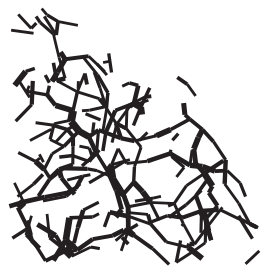

Zone 1

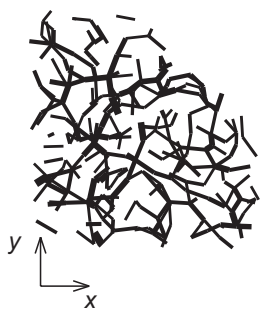

(b)

Fig. 11. Network of contact forces in three zones for monodisperse specimen (only forces exceeding mean force plus one standard deviation are illustrated): (a) $\varepsilon_{\mathrm{a}}=6 \cdot 2 \%$ (around peak); (b) $\varepsilon_{\mathrm{a}}=12 \cdot 3 \%$ (post peak)

Each contact force is represented by a line segment connecting the centroids of two contacting particles, with the line width being proportional to the magnitude of the normal contact force. For ease of visualisation, only contacts where the magnitude of the contact force exceeds the average contact force plus one standard deviation are plotted.

As illustrated in the $x-z$ (vertical) view in Fig. 11, the contact force network is orientated mostly vertically, reflecting the vertical orientation of $\sigma_{1}$. Referring to the plan view of the contact force network in various vertical zones, it is observed that there are a larger number of contact forces with a significant horizontal component in the zone close to the bottom boundary (zone 1) than in the middle zone (zone $3)$. As the average horizontal force is similar in both zones, it can be concluded that there is a smaller number of larger forces in the middle zone. It is also apparent that the continuous strong force chains transmitting the deviatoric load through the specimen tend to be focused towards the centre of the specimen.

The magnitude and the direction of the contact forces in three dimensions can be appreciated more clearly by reference to the two orthogonal views of the contact force vectors in the three zones, as shown in Fig. 12. Again, only contacts where the magnitude of the contact force exceeds the average contact force plus one standard deviation are plotted. The elliptical plots in the $x-z$ views reflect the higher vertical stress than the horizontal stress, and the nearly circular plots in the $x-y$ view reflect the constant confining pressure applied in the radial direction. A slight nonuniformity of the contact forces along the elevation can be observed from Fig. 12. Considering the $z-x$ views, there are a smaller number of forces orientated along the horizontal $(x-y)$ plane in the middle zone (zone 3$)$ than in the zone close to the boundary (zone 1). The contact force network for the specimen with frictionless top and bottom boundaries at an axial strain of $6 \cdot 2 \%$ is also illustrated in Fig. 13. A
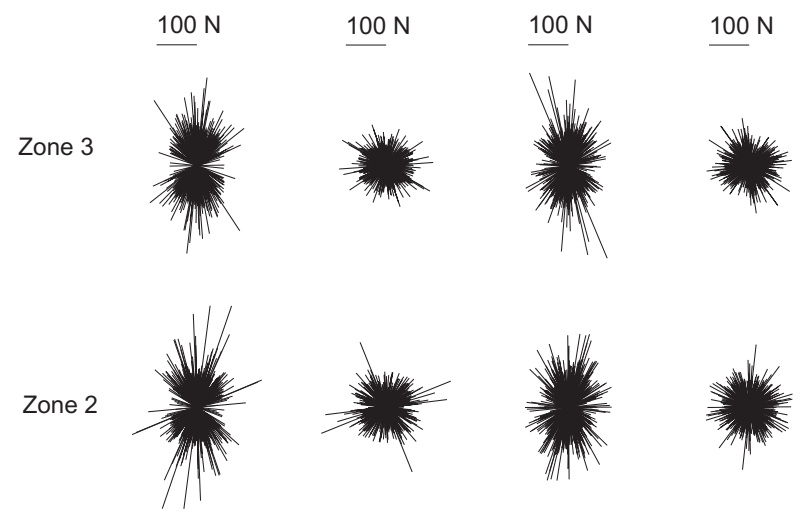

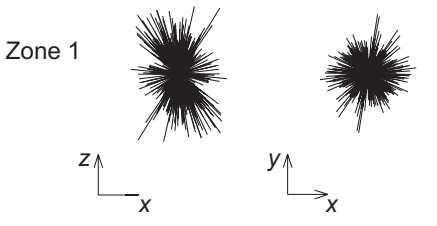

(a)

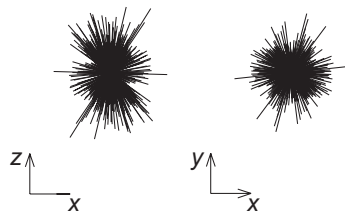

(b)
Fig. 12. Distribution of contact force magnitude and orientation in three zones for monodisperse specimen (only forces exceeding mean force plus one standard deviation are illustrated): (a) $\varepsilon_{\mathrm{a}}=$ $6 \cdot 2 \%$ (around peak); (b) $\varepsilon_{\mathrm{a}}=\mathbf{1 2 \cdot 3 \%}$ (post peak) 
$500 \mathrm{~N}$

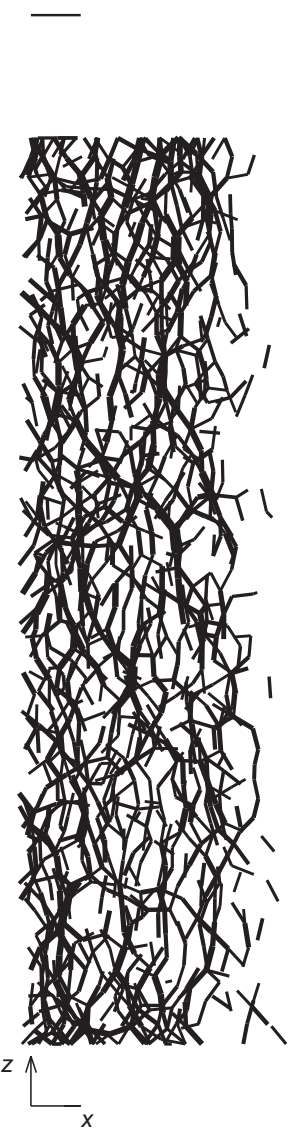

$500 \mathrm{~N}$

Zone 3

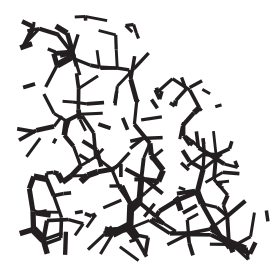

Zone 2

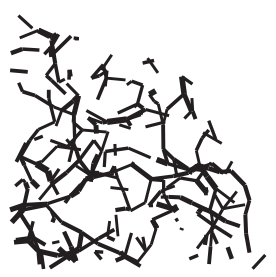

Zone 1

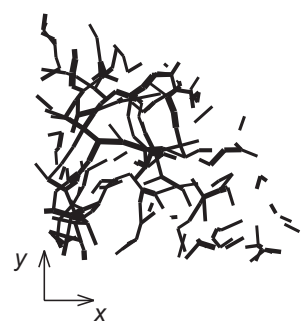

Fig. 13. Distribution of contact force magnitude and orientation in three zones for monodisperse specimen in simulation with frictionless top and bottom boundaries (only forces exceeding mean force plus one standard deviation are illustrated): (a) $\varepsilon_{\mathrm{a}}=$ $6 \cdot 2 \%$ (around peak)

comparison of Fig. 11(a) and Fig. 13 indicates that the difference in the contact network between the frictional boundaries and the frictionless boundaries is less obvious than would be expected, given the well-accepted influence of the boundary friction on the specimen response in triaxial testing.

For the specimen with the rigid circumferential boundaries, the networks of contact forces during the simulation on a monodisperse specimen with $f_{\text {bd }}=0$ and $f_{\text {bd }}=0.228$ are plotted in Fig. 14. By comparing Fig. 14 and Fig. 11, it can be seen that there are more contacts with a finite component normal to the boundary along the periodic boundaries than along the rigid boundaries. As illustrated in Fig. 14(b), there are very strong contact forces along the rigid frictional boundaries below the top boundary and above the base boundary. The plots of the contact forces in the $x-$ $y$ plane clearly demonstrate that the rigid boundaries disturb the network of horizontal forces in the $x-y$ plane, while the periodic boundaries allow a natural and continuous particleparticle contact network to develop in this plane.

\section{Evolution of fabric and coordination number}

The deviator fabric is an indicator of anisotropy of the microstructure (e.g. Cui \& O'Sullivan, 2006). The evolution of both the deviator fabric and the coordination number for the three zones indicated in Fig. 10 is illustrated in Fig. 15 for both monodisperse and polydisperse specimens. The nonuniformity of the specimen and the effect of end restraint are confirmed again by considering the structure and con- tacts within the specimen. It can be observed that both the deviator fabric (anisotropy) and the coordination number in the end zone are obviously smaller than in the middle zones. Considering the coordination number, it is interesting to note that, while there is little variation of the global coordination above axial strains of about $2.5 \%$, the coordination number within the specimen is varying. For the monodisperse specimen the number of contacts in zone 3 increases while the number of contacts in zone 2 decreases during straining, whereas for the polydisperse specimen the coordination number within zone 2 remains approximately constant, and the coordination numbers within zone 1 and zone 3 vary during shearing. The coordination number $N$ within the polydisperse specimen is lower than that within the monodisperse specimen, even though the initial void ratio $\left(e_{0}\right)$ for the polydisperse specimen is slightly lower, as illustrated in Table 1. The coordination number of the smaller spheres is lower than that of the larger spheres.

\section{Analysis of local strains}

One advantage of discrete element modelling is that the internal strains, which cannot be easily observed in the physical tests, can be analysed. A vertical zone inside the specimen with a thickness of $10 \mathrm{~mm}$, zone 1 , as illustrated in Fig. 16, was selected to examine the distribution of strains within the specimen. The strains were calculated using the non-linear homogenisation approach proposed by O'Sullivan et al. (2003). The shear strain $\left|\varepsilon_{z \text {-rad }}\right|$, the volumetric strain $\varepsilon_{\mathrm{vol}}$, the radial strain $\varepsilon_{\mathrm{rad}}$, and the vertical strain $\varepsilon_{\mathrm{Z}}$ in zone 1 for the increment in axial strain from $0 \%$ to $12.3 \%$ are illustrated in Fig. 17(a). Note that for ease of visualisation the magnitude of the shear strain is considered here, $\left|\varepsilon_{z \text {-rad }}\right|$. As shown in Fig. 17(a), all the strain values considered have maximum values close to the specimen mid-height. The positive values of $\varepsilon_{\mathrm{vol}}$ indicate that the specimen is dilating following shearing, and the dilation is localised at the mid-height. The positive values of $\varepsilon_{\text {rad }}$ reflect the motion of the particles radially outwards, as discussed above. While the global average vertical strain is $-12 \cdot 3 \%$, there are also zones with positive vertical strains, indicating the erratic rearrangements of particles within the specimen. The figures indicate that there are two localisations within the specimen; similar complementary localisations have been observed in earlier two-dimensional biaxial DEM simulations (e.g. O'Sullivan et al., 2003). Neither localisation appears to be dominant.

Image-analysis-based techniques have been used by some researchers (e.g. Rechenmacher \& Finno, 2004; Sachan et al., 2006) to analyse local strains in experiments. Such analyses are based on an analysis of the displacements of the particles on the exterior of the specimen. Therefore in the current study, plots of the shear strain $\left|\varepsilon_{\text {z-circ }}\right|$, the volumetric strain $\varepsilon_{\mathrm{vol}}$, the radial strain $\varepsilon_{\text {circ }}$, and the vertical strain $\varepsilon_{\mathrm{z}}$ for the axial strain increment from $0 \cdot 0 \%$ to $12 \cdot 3 \%$ were developed, considering the particles along the exterior of the specimen (i.e. the particles in zone 2 are illustrated in Fig. 17(b)). The shear strain is localised at the mid-height. The distinct localisations observed by Rechenmacher \& Finno (2004) and Sachan et al. (2006) in their experimental studies are not observed, as the material differs from the dense dilative soils discussed in these other works. The $\varepsilon_{\mathrm{vol}}$ values indicate that there is both compression and dilation of the material along the exterior of the sample. The vertical strain localisation was also observed on the surface of the specimen.

To complete the study of the strain non-uniformity within the specimen, plots of the particle rotations were also generated. Earlier two-dimensional studies, including Iwashita \& 


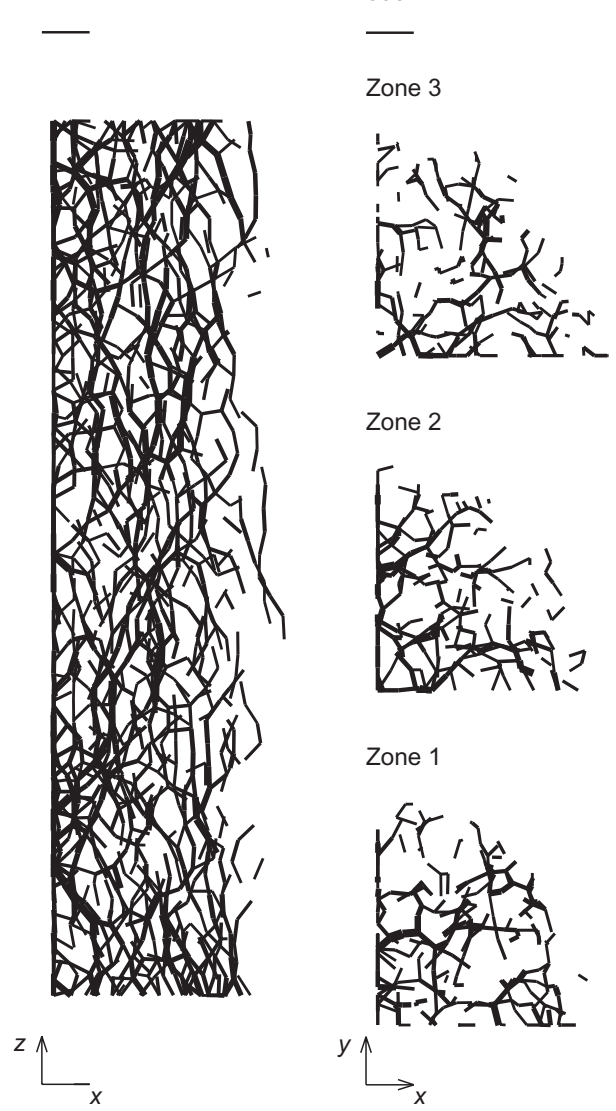

(a)

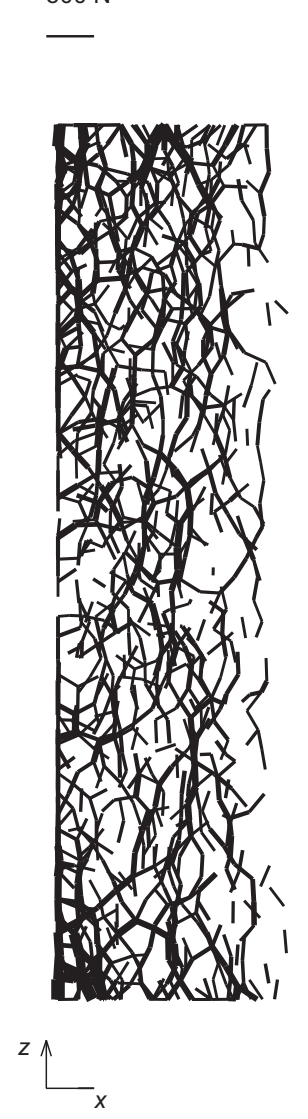

Zone 3

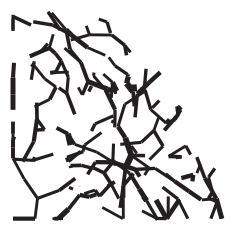

Zone 2

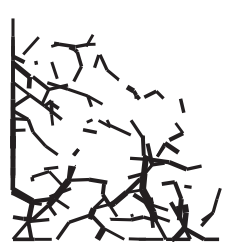

Zone 1

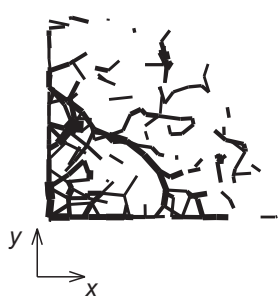

(b)

Fig. 14. Comparison of contact force network for rigid boundary condition $\left(\varepsilon_{\mathrm{a}}=\mathbf{6 \cdot 2 \%}\right)($ only forces exceeding mean force plus one standard deviation are illustrated; monodisperse specimen): (a) $f_{\text {bd }}=0 \cdot 0$; (b) $f_{\text {bd }}=0 \cdot 228$

Oda (2000) and O’Sullivan et al. (2003), have demonstrated that significant particle rotations occur along localisations, especially for circular particles. Fig. 18 illustrates the total particle rotations for both the specimen types for the axial strain increment from $0 \cdot 0 \%$ to $12 \cdot 3 \%$. Noting that visualisation of rotations in three dimensions is non-trivial, in all cases the rotation around an axis orthogonal to the plane of projection is considered, the size of the circle plotted is proportional to the magnitude of the rotation, and a filled circle indicates clockwise rotation, while an empty circle indicates counter-clockwise rotation. The results confirm the earlier two-dimensional observations, that is, that the maximum rotations and maximum strains occur at the same locations. It is difficult, however, to identify any trend in the orientation of the rotations, in contrast to the findings of earlier two-dimensional DEM studies (e.g. O'Sullivan, 2002). The particle rotations in the polydisperse specimen tend to be higher, as illustrated in Fig. 18, because the smaller particles tend to have a lower coordination number and consequently have less resistance to rotation.

\section{CONCLUSIONS}

This paper has proposed a new, computationally efficient approach to modelling axisymmetrical problems using DEM. This new approach uses circumferential periodic boundaries, and a triangulation-based stress-controlled membrane. Validation was achieved by simulating a series of physical triaxial tests on specimens of steel spheres under vacuum confinement. A sensitivity analysis demonstrated that these circumferential periodic boundaries maintained a continuous network of particle-particle contacts within the specimen, allowing the three-dimensional material response to be captured accurately. The use of a system of circumferential rigid walls to model axisymmetric problems using DEM was shown to be inappropriate.

A detailed analysis of the micro-scale parameters using the DEM modelling results illustrated the non-uniformity of the stresses, the interparticle contact forces, the fabric, and the strains within the triaxial specimen. The peak stress ratio at the specimen mid-height exceeds the peak stress ratio of the material close to the boundaries. In addition, as the strong force chains tend to be located closer to the centre of the specimen, the peak stress ratio calculated for the interior volumes tends to exceed the peak stress ratio as calculated from the boundary forces. A smaller number of large interparticle contact forces developed close to the specimen mid-height in comparison with the networks close to the boundaries. While the overall coordination number stabilised at strain values exceeding about $3 \cdot 0 \%$, the coordination number within the specimen was not constant, with some regions experiencing an increase in coordination number and some regions experiencing a decrease in coordination number. The anisotropy of the material also tended to be greater, further away from the specimen boundaries. An analysis of the local strains indicated that measurements of the particle displacements on the exterior of the specimen did not give a clear picture of the internal strain distribution, for the relatively loose material considered here. While a correlation between the strains and the particle rotations was observed, no clear trend in the particle rotations was noted. 


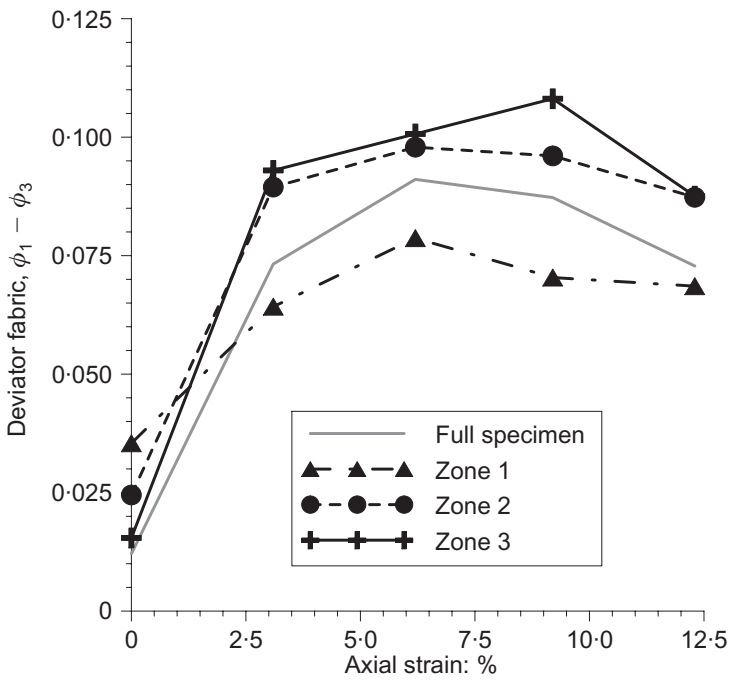

(a)

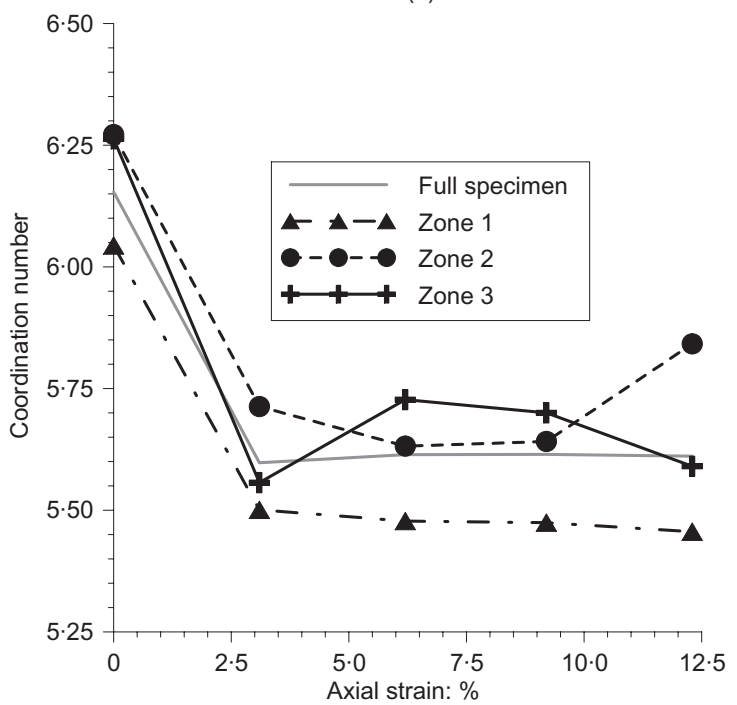

(c)

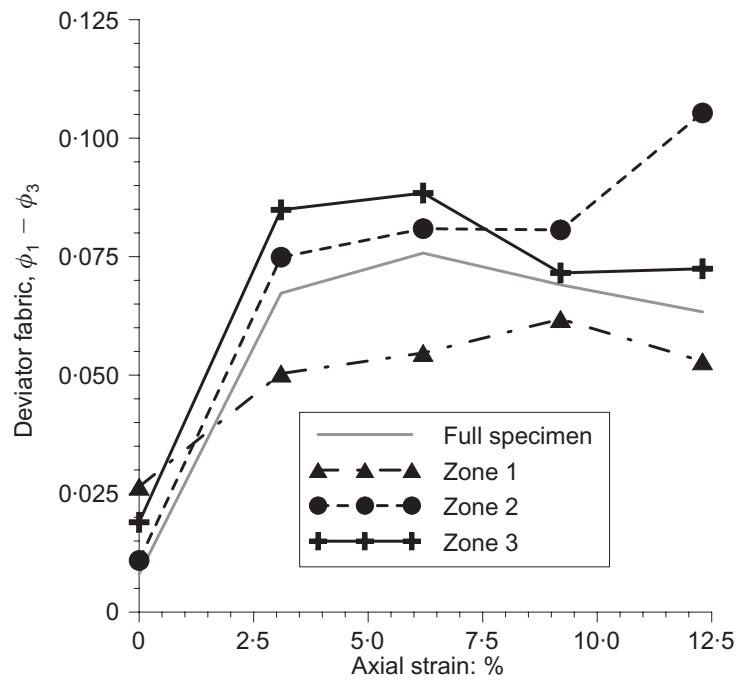

(b)

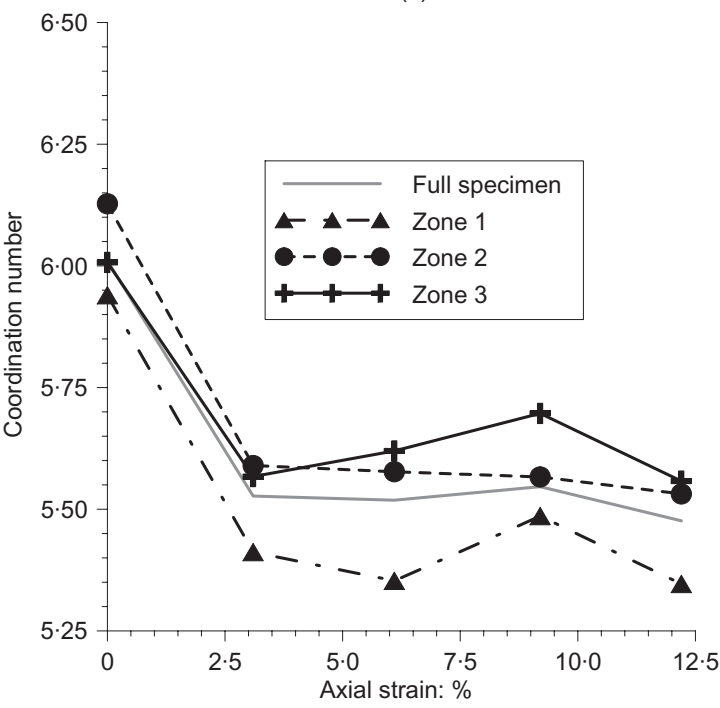

(b)

Fig. 15. Evolution of deviator fabric and coordination number in various zones: (a) deviator fabric against axial strain for monodisperse specimen; (b) deviator fabric against axial strain for polydisperse specimen; (c) coordination number against axial strain for monodisperse specimen; (d) coordination number against axial strain for polydisperse specimen

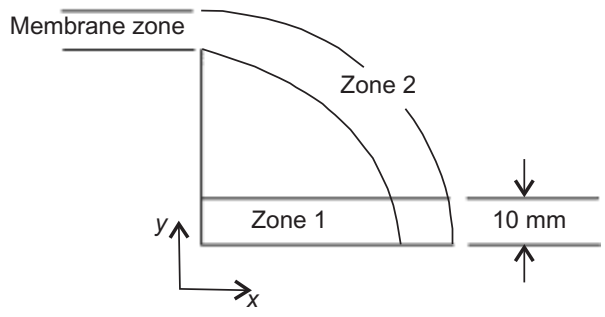

Fig. 16. Illustration of zones selected for strain calculation
The simple granular material used in this study differs from real soil, and was selected to enable close coupling of the numerical simulations and physical tests. If a more complex granular material had been used, the quality of the validation would have been compromised. The results presented here give a qualitative insight into the micromechanics of sand response in the triaxial apparatus. More quantitative information about the micromechanics of real soil response can be achieved in the future by incorporating more realistic particle geometries in the DEM model. How-

Table 1. Comparison of coordination number $N$ and void ratio $e$ for monodisperse and polydisperse specimens

\begin{tabular}{|c|c|c|c|c|c|c|c|}
\hline \multirow[t]{3}{*}{$\varepsilon_{\mathrm{a}}: \%$} & \multicolumn{2}{|c|}{ Monodisperse specimen } & \multicolumn{5}{|c|}{ Polydisperse specimen } \\
\hline & \multirow[t]{2}{*}{$e$} & \multirow[t]{2}{*}{$N$} & \multirow[t]{2}{*}{$e$} & \multicolumn{4}{|c|}{$N$} \\
\hline & & & & All spheres & $r=2 \mathrm{~mm}$ & $r=2.5 \mathrm{~mm}$ & $r=3 \mathrm{~mm}$ \\
\hline 0 & $0 \cdot 615$ & $6 \cdot 22$ & $0 \cdot 604$ & $6 \cdot 10$ & 4.69 & 5.99 & $7 \cdot 40$ \\
\hline $3 \cdot 1$ & 0.567 & 5.67 & 0.557 & $5 \cdot 64$ & $4 \cdot 41$ & $5 \cdot 53$ & $6 \cdot 77$ \\
\hline $6 \cdot 1$ & 0.563 & 5.69 & $0 \cdot 562$ & 5.59 & $4 \cdot 28$ & 5.49 & $6 \cdot 73$ \\
\hline $9 \cdot 2$ & $0 \cdot 584$ & 5.69 & 0.570 & $5 \cdot 53$ & $4 \cdot 18$ & 5.45 & $6 \cdot 70$ \\
\hline $12 \cdot 2$ & 0.589 & $5 \cdot 68$ & 0.569 & 5.57 & $4 \cdot 24$ & $5 \cdot 46$ & $6 \cdot 71$ \\
\hline
\end{tabular}



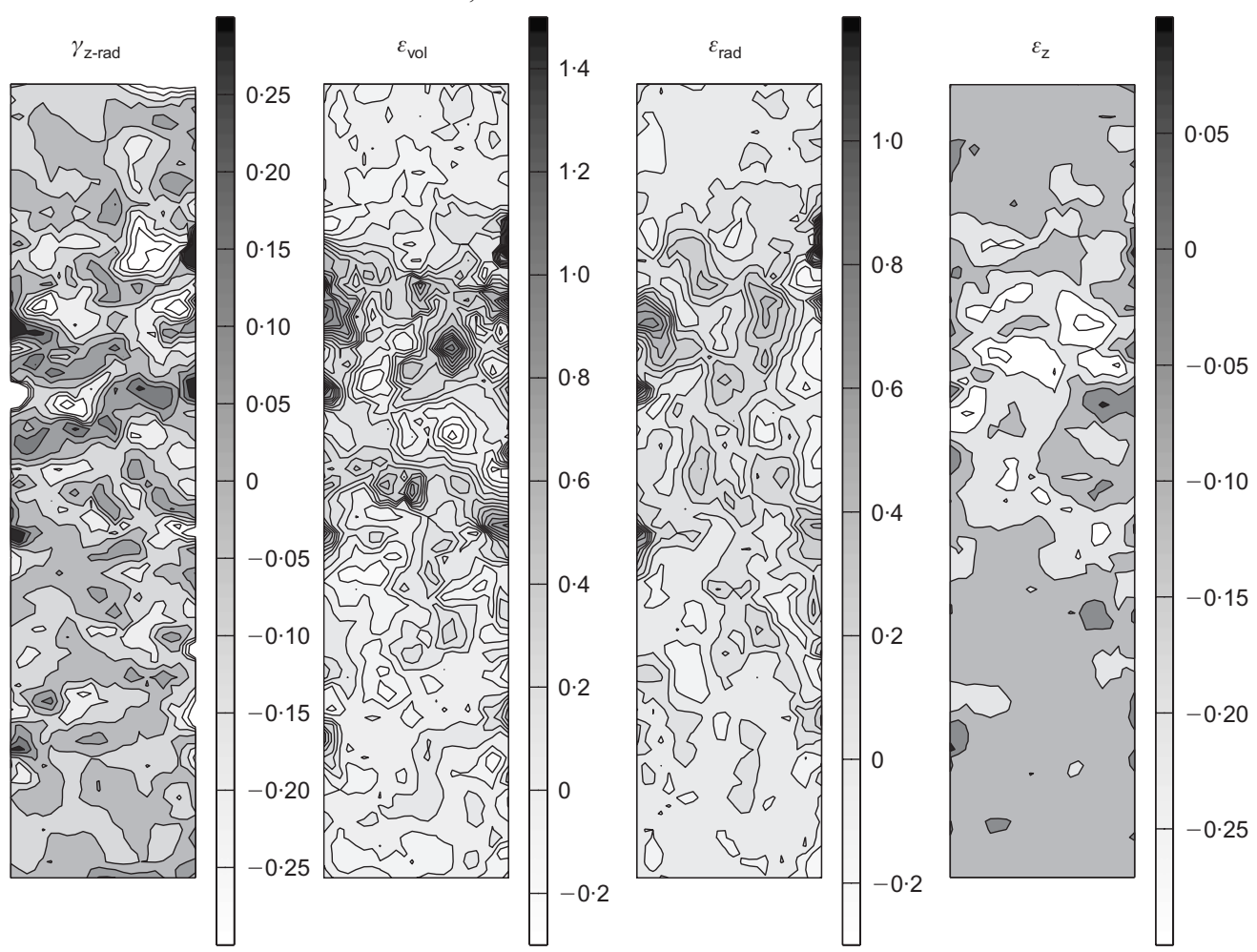

(a)

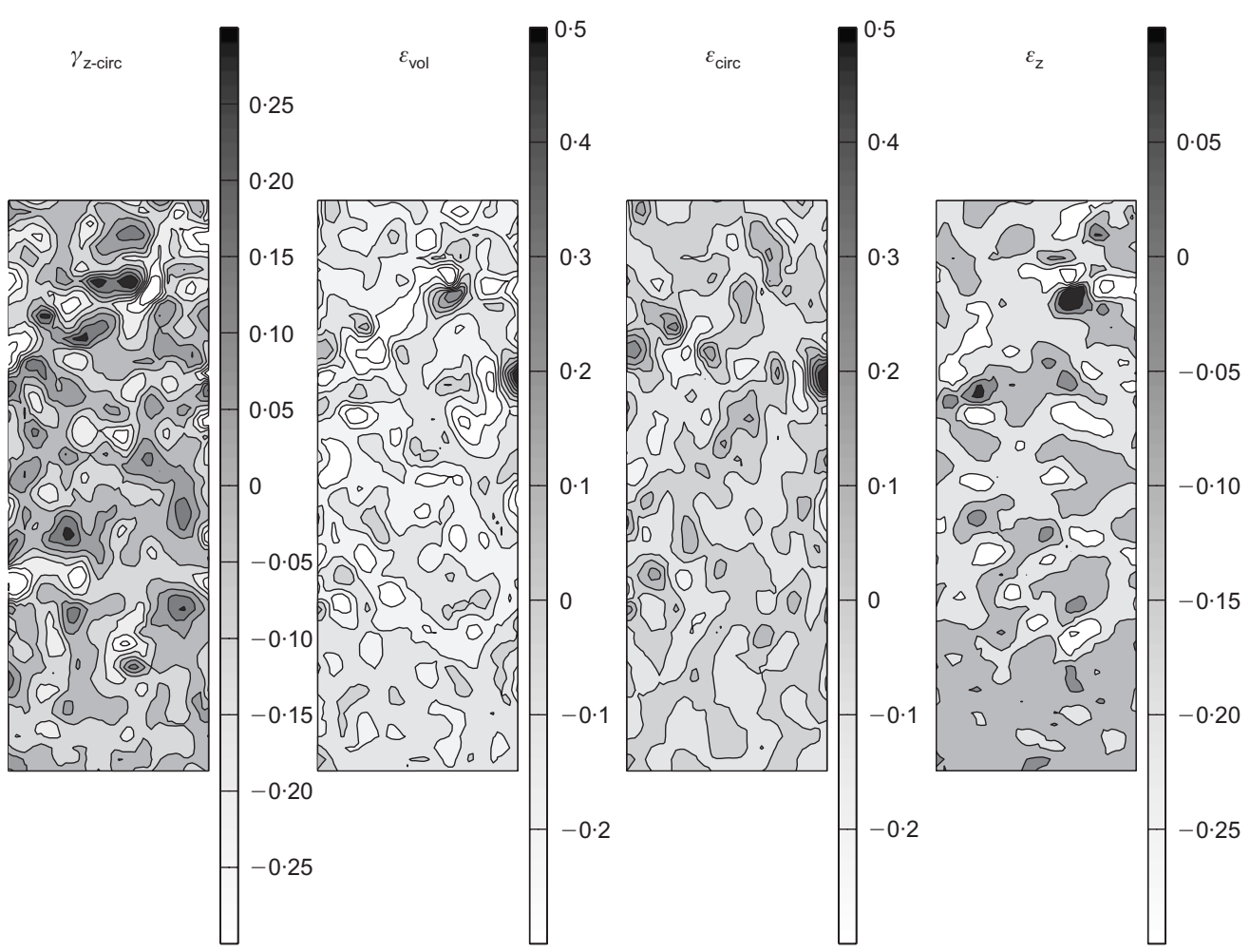

(b)

Fig. 17. Strain localisation in monodisperse triaxial specimen for $\varepsilon_{\mathrm{a}}$ from $0 \%$ to $12 \cdot 3 \%$ : (a) zone 1 ; (b) zone 2

ever, prior to incorporating the complexity of real soil in DEM simulations, it is important to demonstrate the accuracy of the numerical models and the appropriateness of the boundary conditions using simple granular materials such as those used in this study.

\section{ACKNOWLEDGEMENTS}

Funding for this research was provided by the Irish Research Council for Science, Engineering and Technology (IRCSET) under the Basic Research Grant Scheme. Additional funding was provided under the UCD Presidents 


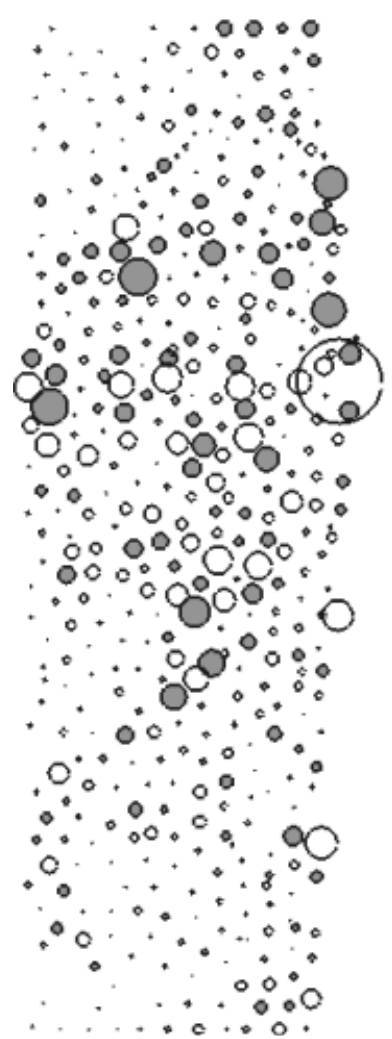

(a)

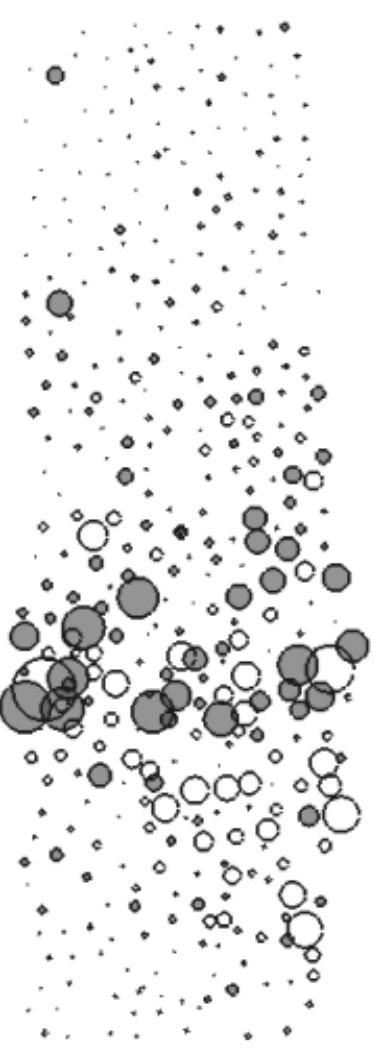

(b)
Fig. 18. Particle rotations in zone 1 for $\varepsilon_{\mathrm{a}}$ from $0 \%$ to $12 \cdot 3 \%$ : (a) monodisperse specimen; (b) polydisperse specimen

Research Award Grant Scheme. The authors are grateful to Mr George Cosgrave, University College Dublin, for his assistance in performing the laboratory tests. Completion of this research was also made possible by the support of Dr Mike Long, University College Dublin.

$\begin{aligned} \text { NOTATION } & \\ e & \text { void ratio } \\ f_{\mathrm{bb}} & \text { interparticle friction coefficient } \\ \mathrm{f}_{\mathrm{bd}} & \text { particle-boundary friction coefficient } \\ l_{i}^{\mathrm{c}} & \text { branch vector } \\ f_{j}^{\mathrm{c}} & \text { contact force vector } \\ N & \text { coordination number } \\ N c & \text { number of contacts } \\ r & \text { Radius of particle } \\ \mathrm{V} & \text { volume of measurement region } \\ \varepsilon_{a} & \text { Axial strain } \\ \varepsilon_{\mathrm{circ}} & \text { cirumferential strain } \\ \varepsilon_{\text {rad }} & \text { radial strain } \\ \varepsilon_{\mathrm{z}} & \text { vertical strain } \\ \varepsilon_{\mathrm{vol}} & \text { volumetric strain } \\ \phi^{\prime}, \phi_{\text {peak }} & \text { angle of shearing resistance, peak angle of shearing } \\ & \text { resistance } \\ \gamma_{\mathrm{z} \text {-rad }}, \gamma_{\mathrm{z}-\mathrm{circ}} & \text { shear strain (cylindrical coordinates) } \\ \theta & \text { angle between two circumferential periodic } \\ & \text { boundaries } \\ \sigma_{1} & \text { major principal stress } \\ \sigma_{3} & \text { minor principal stress } \\ \sigma_{\mathrm{ij}} & \text { average stress tensor }\end{aligned}$

\section{REFERENCES}

Clayton, C. R. I. \& Khatrush, S. A. (1986). A new device for measuring local axial strains on triaxial specimens. Géotechnique 36, No. 4, 593-597.

Cuccovillo, T. \& Coop, M. R. (1997). The measurement of local axial strains in triaxial tests using LVDTs. Géotechnique 47, No. $1,167-171$.

Cui, L. (2006). Developing a virtual test environment for granular materials using discrete element modelling. $\mathrm{PhD}$ thesis, University College Dublin, Ireland.

Cui, L. \& O'Sullivan, C. (2005). Development of a mixed boundary environment for axi-symmetric DEM analyses. Proc. 5th Int. Conf. on Micromechanics of Granular Media, Stuttgart, 1, 301-305.

Cui, L. \& O'Sullivan, C. (2006). Exploring the macro- and microscale response characteristics of an idealized granular material in the direct shear apparatus. Géotechnique 56, No. 7, 455-468.

Cundall, P. \& Strack, O. (1979). A discrete numerical model for granular assemblies. Géotechnique 29, No. 1, 47-65.

Frost, D. \& Yang, C.-T. (2003). Effect of end platens on microstructure evolution in dilatant specimen. Soils Found. 43, No. 4, $1-11$.

Iwashita, K. \& Oda, M. (2000). Micro-deformation mechanism of shear banding process based on modified distinct element method. Powder Technol. 109, Nos 1-3, 192-205.

Jang, D. J. \& Frost, J. D. (2000). Use of image analysis to study the microstructure of a failed sand specimen with a shear band. Can. Geotech. J. 37, No. 5, 1141-1149.

Jardine, R. J., Symes, M. J. \& Burland, J. B. (1984). The measurement of soil stiffness in the triaxial apparatus. Géotechnique 34, No. 3, 323-340.

Jodrey, W. \& Tory, E. (1985). Computer simulation of close random packing of equal spheres. Phys. Rev. A 32, No. 4, 2347-2351.

Klotz, E. U. \& Coop, M. R. (2002). On the identification of critical state lines for sands. ASTM Geotech. Test. J. 25, No. 3, 289302.

Lin, X. \& Ng, T.-T. (1997). A three-dimensional discrete element model using arrays of ellipsoids. Géotechnique 47, No. 2, 319329.

Morchen, N. \& Walz, B. (2003). Model generation and calibration for a pile loading in the particle flow model. In Numerical modelling in micromechanics via particle methods (ed. $\mathrm{H}$. Konietzky), pp. 189-195. Rotterdam: A. A. Balkema.

$\mathrm{Ng}$, T.-T. (2004). Shear strength of assemblies of ellipsoidal particles. Géotechnique 54, №. 10, 659-669.

O'Neill, S. (2005). A fundamental examination of the behaviour of granular media and its application to discrete element modelling. Master's thesis, University College Dublin, Ireland.

O'Sullivan, C. (2002). The application of discrete element modelling to finite deformation problems in geomechanics. $\mathrm{PhD}$ thesis, University of California, Berkeley.

O'Sullivan, C., Bray, J. D. \& Li, S. (2003). A new approach for calculating strain for particulate media. Int. J. Numer. Anal. Methods Geomech. 27, No. 10, 859-877.

O'Sullivan, C., Bray, J. \& Riemer, M. (2004). An examination of the response of regularly packed specimens of spherical particles using physical tests and discrete element simulations. J. Engng Mech., ASCE 130, No. 10, 1140-1150.

Otani, J., Mukunoki, T. \& Obara, Y. (2000) Application of X-ray CT method for characterization of failure in soils. Soils Found. 40, No. 2, 111-118.

Potts, D. M. \& Zdravkovic, L. (1999). Finite element analysis in geotechnical engineering. London: Thomas Telford.

Rowe, P. W. \& Barden, L. (1964). Importance of free ends in triaxial testing. J. Soil Mech. Found. Div. ASCE 90, No. SM1, $1-27$.

Rechenmacher, A. L. \& Finno, R. J. (2004). Digital image correlation to evaluate shear banding in dilative sands. ASTM Geotech. Test. J. 27, No. 1, 13-22.

Sachan, A., Lin, H. \& Penumadu, D. (2006). Use of digital imaging technique for studying strain localizations. Proceedings of GeoCongress 2006, Atlanta, CD-rom.

Shewchuk, J. R. (1999). Lecture notes on Delauney mesh generation. University of California, Berkeley: Department of Electrical Engineering and Computer Science.

Thornton, C. (1979). The conditions for failure of a face-centred 
cubic array of uniform rigid spheres. Géotechnique 29, No. 4, $441-459$.

Thornton, C. (2000). Numerical simulations of deviatoric shear deformation of granular media. Géotechnique 50, No. 1, 43-53.
Tsunekawa, H. \& Iwashita, K. (2001). Numerical simulation of triaxial test using two and three dimensional DEM. In Powders and grains 01 (ed. Y. Kishino), pp. 177-182. Rotterdam: A. A. Balkema. 